\title{
Prosthesis retention after an infected hip prosthesis: hip fractures versus primary total hip prosthesis, data from $1998-2015$
}

\author{
Lieke M.A. de Vries ${ }^{\bowtie}$, William C. Neve, Jeroen Steens \\ Westfriesgasthuis, Department of Orthopaedic surgery, Maelsonstraat 3, 1624 NP Hoorn, The Netherlands. \\ $\triangle$ Corresponding author: Lieke M.A. de Vries, Orthopaedic research coordinator, Westfriesgasthuis, Department of Orthopaedic surgery, Maelsonstraat 3, 1624 \\ NP Hoorn, The Netherlands. +31 (0)299 855562; L.M.A.deVries@westfriesgasthuis.nl \\ (c) Ivyspring International Publisher. This is an open access article distributed under the terms of the Creative Commons Attribution (CC BY-NC) license \\ (https://creativecommons.org/licenses/by-nc/4.0/). See http://ivyspring.com/terms for full terms and conditions.
}

Received: 2017.11.20; Accepted: 2018.05.04; Published: 2018.06.10

\begin{abstract}
Background: A prosthetic joint infection (PJI) is a serious complication and specifically a burden for patients after hip fracture surgery, as they are mostly frail elderly patients with multiple comorbidities. Since treatment protocols are lacking there is a need to evaluate current practice.

Aim: To evaluate the difference in prosthesis retention after an infected primary total hip replacement (THR) compared to PJI after hip prosthesis surgery performed for a hip fracture.

Methods: We retrospectively collected data of patients who developed PJI after primary THR or after hip fracture surgery (THR or hemiarthroplasty) in the Westfriesgasthuis Hospital between 1998 and 2015. Main outcome variables were DAIR treatment and prosthesis retention.

Findings: A PJI developed in 48 patients after primary THR and in 23 patients after hip fracture surgery. DAIR was performed in all patients after primary THR and in $87.0 \%$ of patients after hip fracture surgery $(p<0.05)$. In $11.4 \%$ of patients after primary THR, revision surgery was performed within 1 year after PJI compared to $34.8 \%$ after hip fracture surgery $(p<0.05)$. Only $2.1 \%$ of patients deceased within 1 year after infection of primary THR compared to $34.8 \%$ after hip fracture surgery $(p<0.05)$.

Conclusion: Our results showed that prosthesis retention in patients with a PJI after hip fracture surgery is $23 \%$ lower than in patients with a PJI after primary THR. This is probably due to the fact that patients who experience a hip fracture are mostly frail elderly with multiple comorbidities and therefore less able to conquer a PJI.
\end{abstract}

Key words: prosthetic joint infection; primary hip prosthesis; hip fracture surgery; frail elderly; survival rate.

\section{Introduction}

A prosthetic joint infection (PJI) is a serious complication which requires extensive treatment, consisting of long term antibiotics, debridement surgery and in some cases revision surgery [16]. This is specifically a burden for patients after hip fracture surgery, since they are mostly frail elderly patients with multiple comorbidities. It is important to maintain optimal quality of life and eradicate the infection, while multiple surgeries should be avoided to minimize surgical risks.
In 2013, delegates from 52 countries participated in an international consensus meeting regarding current practice for PJI management [16]. This management consists of debridement, antibiotics, irrigation and retention of the prosthesis (DAIR) and/or eventually revision surgery. Nevertheless, this consensus meeting was limited to primary hip and knee arthroplasty. Treatment protocols regarding PJI after hip fracture surgery are lacking, though there is a clear difference with patients after primary total hip 
replacement (THR): they have different baseline features due to the acute character of a hip fracture compared to the elective procedure for THR, like advanced age and comorbidities but also the acute stress and inflammatory states resulting from the fracture $[5,10]$. PJIs after hip fracture surgery are mostly early post-surgical, while among primary THR the percentage of late-chronic infections is higher (higher proportion of haematogenous infections) [10]. Furthermore, treatment of a PJI in this acute patient group requires a different approach since these frail elderly patients have a higher risk of complications [15]. To finalize these guidelines, evaluation of the current treatment practice is needed.

The aim of this study was to evaluate the difference in prosthesis retention after PJI in primary THR compared to PJI after hip prosthesis surgery performed for a hip fracture. We hypothesized that prosthesis retention after infection will be higher after primary THR compared to hip fracture surgery. Patients after hip fracture surgery are usually frail elderly patients, more sensitive for complications, and therefore less capable of surviving PJI.

Table 1. Criteria for deep PJI [16]

* Two positive periprosthetic cultures with phenotypically identical organisms, or

* A sinus tract communicating with the joint, or

* A combination of three of the following minor criteria:

- Elevated serum C-reactive protein (CRP) AND erythrocyte sedimentation rate (ESR)

- Elevated synovial fluid white blood cell (WBC) count OR ++change on leukocyte esterase test strip

- Elevated synovial fluid polymorphonuclear neutrophil percentage (PMN\%)

- Positive histological analysis of periprosthetic tissue

- A single positive culture

\section{Methods}

The study population consisted of all patients who developed a PJI within 1 year after primary THR or hip fracture surgery (THR or hemiarthroplasty) in Westfriesgasthuis Hospital between 1998 and 2015. Criteria for a PJI are shown in table 1. Treatment of PJI consisted of DAIR, antibiotic treatment or in some cases revision surgery. The choice of treatment was based on local protocols considering the clinical presentation of the patient like wound inspection, acute or chronic presentation of symptoms, presence of a fever and infection parameters. This treatment protocol did not change between 1998 and 2015. DAIR consisted of an open procedure without component exchange. The choice for a second and/or third DAIR procedure was based on the clinical features of the patient, the infection parameters and wound inspection. Data for this study were retrospectively collected from the hospital electronic databases and included patient characteristics (age, gender,
American Society of Anesthesiologists classification (ASA), and body mass index (BMI)), the time from surgery till infection, frequency of DAIR and revision surgery and mortality within 1 year after infection. Micro-organisms were identified according to standard local protocols. Results were analysed and compared to literature.

Data management and analysis were performed using SPSS 23.0 (2015). Nominal variables (gender, DAIR, revision surgery and mortality $<1$ year) were analysed with chi square analysis. Ordinal variables (ASA and frequency of DAIR) were analysed with the Mann-Whitney test. Continuous variables (age, BMI and time from surgery till infection) were analysed with the t-test. Comparisons between the two groups were made using unrelated t-tests and chi square analysis. A successful treatment was defined as prosthesis retention within 1 year after PJI. A survival analysis was performed to test for significant changes in prosthesis retention between the two groups. A p-value $<0.05$ was considered significant. No ethical approval was necessary for this study.

\section{Results}

A PJI developed in 48 out of 5138 patients $(0.9 \%)$ after primary THR and in 23 out of 1457 patients $(1.6 \%)$ after hip fracture surgery (hemiarthroplasty or THR), therefore 71 patients were included in this study. Patients' characteristics are presented in table 2. Patients after hip fracture surgery were significantly older than patients after primary THR (80.2 vs 71.1 years old, respectively). DAIR was performed in all patients after primary THR and in $87.0 \%$ of patients after hip fracture surgery $(\mathrm{p}<0.05)$. In the remaining $13.0 \%$ of the patients, acute revision surgery was chosen without prior DAIR procedure. This was due to repeated dislocation of the hip prosthesis in all three cases.

In both groups, the most frequent microorganisms associated with PJI were Staphylococcus aureus and coagulase negative staphylococcus (CNS), figure 1. There was no significant difference in detected micro-organisms between both groups. Furthermore, in $10.4 \%$ of patients after primary THR, revision surgery was performed within 1 year after PJI compared to $34.8 \%$ after hip fracture surgery $(p<0.05)$, table 2 . Figure 2 shows prosthesis survival until 1 year after in PJI in primary THR $(89.6 \%)$ versus hip fracture surgery $(65.2 \%)$. When the survival analysis was corrected for age and ASA using cox-regression analysis, the same difference was found between both groups. Only 2.1\% of patients deceased within 1 year after infection compared to $34.8 \%$ after infection after hip fracture surgery $(\mathrm{p}<0.05)$. 


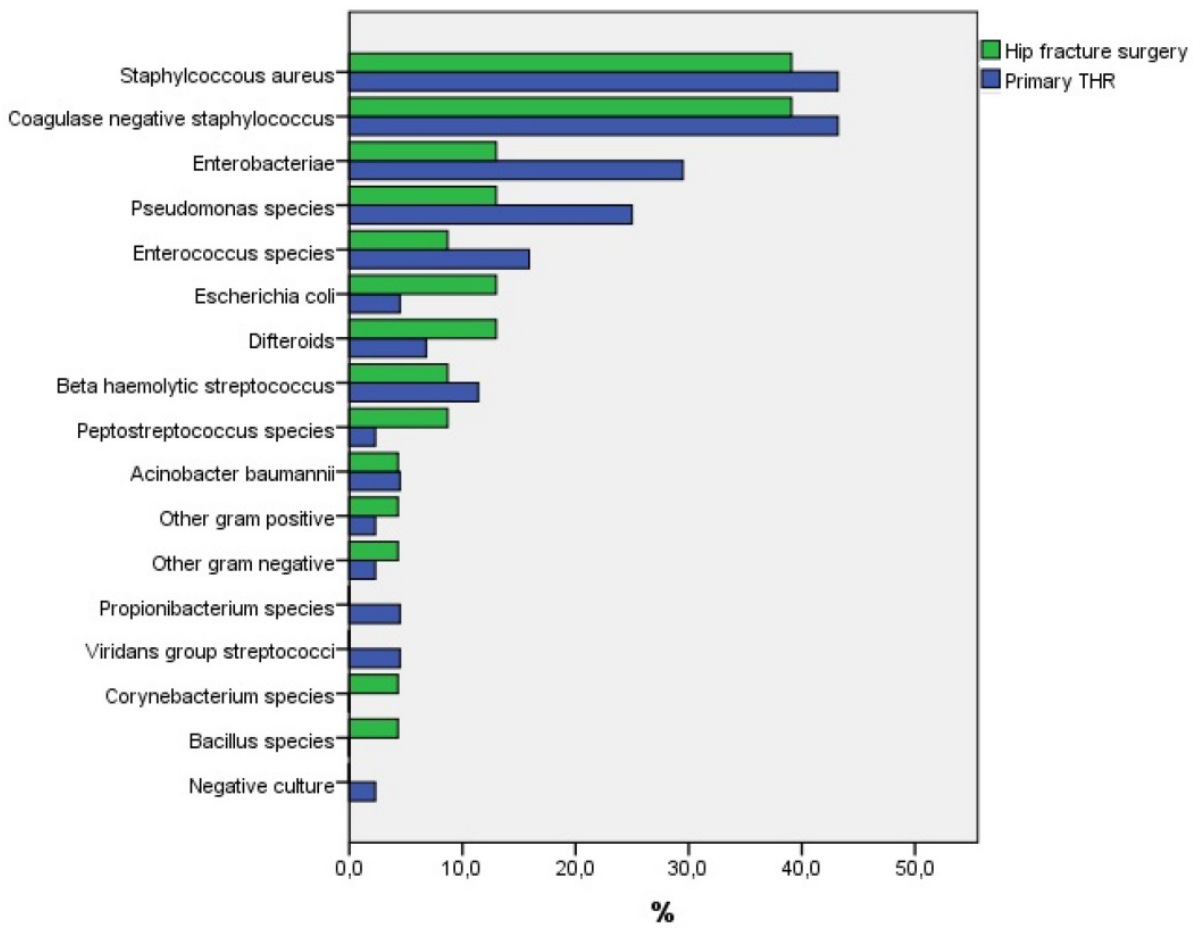

Figure 1. Percentage micro-organisms associated with PJI in primary THR and after hip fracture surgery

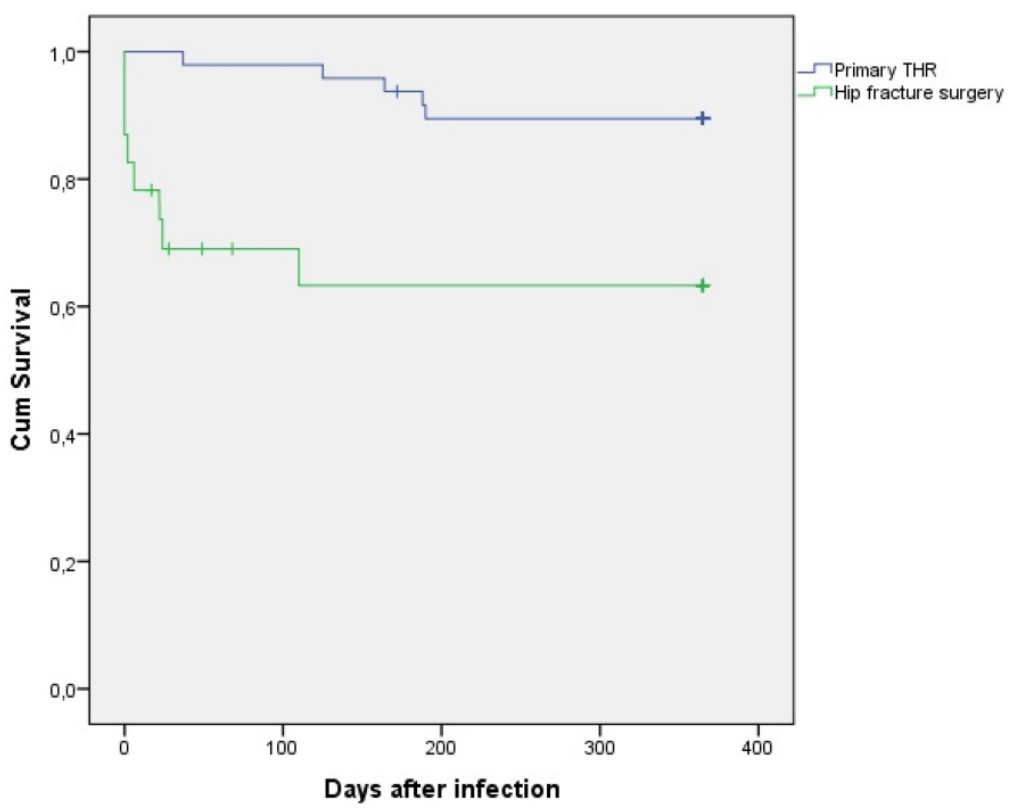

Figure 2. Prosthesis survival until 1 year after infection: primary THR versus hip fracture surgery

Table 2. Characteristics of patients

\begin{tabular}{|c|c|c|c|}
\hline & Primary $\operatorname{THR}^{\times}(\mathrm{n}=48)$ & Hip fracture $(n=23)$ & P value \\
\hline Gender $(\mathrm{M} / \mathrm{F})$ & $11 / 37$ & $5 / 18$ & 0,91 \\
\hline Age (mean (range)) & $71.1(54-86)$ & $80.2(58-93)$ & 0,00 \\
\hline $\mathrm{BMI}(\text { mean }(\mathrm{sd}))^{*}$ & $30.8(6,7)$ & $26.8(3,4)$ & 0,09 \\
\hline ASA $(\text { median }(\text { range }))^{* *}$ & $2(1-3)$ & $3(1-4)$ & 0.01 \\
\hline Time primary surgery till infection (days (range)) & $26.8(6-92)$ & $35.7(5-238)$ & 0,30 \\
\hline DAIR performed $(\mathrm{N}(\%))^{* * *}$ & $48(100.0 \%)$ & $20(87.0 \%)$ & 0,01 \\
\hline Frequency of DAIR (median (range)) & $1(1-3)$ & $1(0-3)$ & 0,14 \\
\hline Revision surgery performed $<1$ year after infection $(\%)$ & $5(10.4 \%)$ & $8(34.8 \%)$ & 0,01 \\
\hline Patients deceased $<1$ year after infection $(\%)$ & $1(2.1 \%)$ & $8(34.8 \%)$ & 0,00 \\
\hline
\end{tabular}

*BMI: Body Mass Index = weight $(\mathrm{kg}) /(\text { height }(\mathrm{m}))^{2}$

**ASA classification $=$ American Society of Anesthesiologists classification

${ }^{* * *}$ DAIR: Debridement, Antibiotics, Irrigation and Retention

xTHR: Total Hip Replacement 


\section{Discussion}

The aim of this study was to evaluate the difference in prosthesis retention after an infected THR compared to PJI after hip prosthesis surgery performed for a hip fracture. The results showed that prosthesis survival was significantly lower after PJI in hip fracture patients compared to primary THR (65.2\% vs $89.6 \%$ respectively). This could be explained by the fact that patients with a hip fracture are mostly frail elderly with multiple comorbidities and therefore less capable of recovering from a PJI $[5,20]$. This also resulted in higher mortality rates in this fragile patient group (34.8\% after hip fracture surgery vs $2.1 \%$ after primary THR) as mentioned in other studies [6, 12, 19]. Furthermore, the acute situation of a hip fracture is not an ideal starting point for optimal recovery. Most patients are over 70 years old and have pre-existing medical comorbidities which could lead to higher morbidity and mortality rates [3]. Though optimal treatment protocols do not exist yet for PJI after hip fracture surgery, most hospitals use their own local protocol based on expert opinion and other available guidelines in which DAIR is often the first treatment option in early infections [16]. The success rate of DAIR for PJI after primary THR described in literature varies between $41-82 \%$ [4, 8, 9, 11, 19]. Mellner et al. [11] described 28 patients with a PJI after a femoral neck fracture (hemiarthroplasty or THR) in which DAIR was used. They found a success rate of $82 \%$ after DAIR yet with a long median follow up of 31 months. They also confirmed the favourable treatment option of DAIR in this fragile patient group. Lora-Tamayo et al. [10] compared different hip devices in patients with a PJI: hip hemiarthroplasty (HHA) or total hip arthroplasty (THA). They found that HHA patients had similar odds of being cured and retaining their prosthesis as did those with THA. This unexpected finding was probably due to the higher proportion of haematogenous infections in the THA group which indicated a worse outcome as previously reported. Nevertheless, the THA group consisted of patients with elective hip arthrosis as well as hip fractures. Our study only consists of patients with an acute PJI and confirms the good results for DAIR as a first treatment option for both primary THR as well as hip fracture surgery.

Our results confirm that the most common micro-organisms associated with PJI were staphylococcus aureus and CNS for both primary THR and hip fracture surgery. This was also seen in previous studies $[2,19]$. To minimize the risk of PJI, preoperative screening of nasal colonization of staphylococcus aureus could be used [17, 18]. Many hospitals have implemented this method in their local protocol for elective arthroplasty, though in the acute situation of a hip fracture, where increased waiting time till surgery has an increased risk of developing PJI, it is impossible to implement this [19]. Since frailty and comorbidities increase the risk of surgical complications, further research is necessary to optimize the patient before surgery and therefore decrease as many risks as possible.

To our knowledge, there are limited studies describing a large cohort of patients with a PJI. Our incidence rates of PJI after hip fracture surgery $(1.6 \%)$ and after primary THR $(0.9 \%)$ are relatively low compared to other literature and national surveillance data $(2-17 \%$ vs $1 \%$ respectively) $[1,7,11,13,14,19]$. Since the incidence rates of PJI are very low, it is important to follow-up these patients to evaluate their functional well-being and eventually establish evidence-based treatment protocols.

\section{Conclusion}

Patients with a PJI after hip fracture surgery have higher mortality rates and show less prosthesis retention compared to patients with a PJI after primary THR, which is probably due to the increased frailty and comorbidities. Since these patients differ from patients with a primary THR, it is recommended to take this into account when developing treatment algorithms.

\section{Competing Interests}

The authors have declared that no competing interest exists.

\section{References}

[1] Bergkvist M, Mukka SS, Johansson L, Ahl TE, Sayed-Noor AS, Sköldenberg OG, et al. Debridement, antibiotics and implant retention in early periprosthetic joint infection. Hip Int. 2016;26:138-143.

[2] Blomfeldt R, Kasina P, Ottosson C, Enocson A, Lapidus LJ. Prosthetic joint infection following hip fracture and degenerative hip disorder: a cohort study of three thousand, eight hundred and seven consecutive hip arthroplasties with a minimum follow-up of five years. Int Orthop. 2015;39:2091-2096.

[3] Carpintero P, Caeiro JR, Carpintero R, Morales A, Silva S, et al. Complications of hip fractures: a review. World J Orthop 2014;5:402-411.

[4] De Vries L, van der Weegen W, Neve WC, Das H, Ridwan BU, Steens J. The effectiveness of debridement, antibiotics and irrigation for periprosthetic joint infections after hip and knee arthroplasty. A 15 years retrospective study in two community hospitals in the Netherlands. J Bone Jt Infect. 2016;1:20-24.

[5] del Toro MD, Nieto I, Guerrero F, Corzo J, del Arco A, Palomino J, et al. Are hip hemiarthroplasty and total hip arthroplasty infections different entities? The importance of hip fractures. Eur J Clin Microbiol Infect Dis. 2014;33:1439-1448.

[6] Duckworth AD, Philips SA, Stone O, Moran M, Breusch SJ, Biant LC. Deep infection after hip fracture surgery: predictors of early mortality. Injury. 2012;43:1182-1186.

[7] Gallardo-Calero I, Larrainzar-Coghen T, Rodriquez-Pardo D, Pigrau C, Sánchez-Raya J, Amat C, et al. Increased infection risk after hip hemiarthroplasty in institutionalized patients with proximal femur fracture. Injury. 2016;47:872-876.

[8] Guren E, Figved W, Frihagen F, Watne LO, Westberg M. Prosthetic joint infection - a devastating complication of hemiarthroplasty for hip fracture. Acta Orthop. 2017;88:383- 389.

[9] Kazimoglu C, Yalcin N, Onvural B, Akcay S, Agus H. Debridement, antibiotics, irrigation, and retention (DAIR) of the prosthesis after hip hemiarthroplasty infections. Does it work? Int J Artif Organs. 2015;38:454-460.

[10] Lora-Tamayo J, Euba G, Ribera A, Murillo O, Pedrero S, García-Somoza D, et al. Infected hip hemiarthroplasties and total hip arthroplasties: Differential findings and prognosis. J Infect. 2013;67:536-544. 
[11] Mellner C, Eisler T, Knutsson B, Mukka S. Early periprosthetic joint infection and debridement, antibiotics and implant retention in arthroplasty for femoral neck fracture. Hip Int. 2017;27:349-353.

[12]. Nijland LMG, Karres J, Simons AE, Ultee JM, Kerkhoffs GMMJ, Vrouenraets BC. The weekend effect for hip fracture surgery. Injury. 2017;48:1536-1541.

[13] No authors listed. PREZIES, PREventie van ZIEkenhuisinfecties door Surveillance. http://www.rivm.nl/Onderwerpen/P/PREZIES/Incidentieon derzoek_POWI/Definities. Accessed July 2017.

[14] Noailles T, Brulefert K, Chalopin A, Longis PM, Gouin F. What are the risk factors for post-operative infection after hip hemiarthroplasty? Systematic review of literature. Int Orthop. 2016;40:1843-1848.

[15] Partridge JS, Harari D, Dhesi JK. Frailty in the older surgical patient: a review. Age Ageing. 2012;41:142-147.

[16] Parvizi J and Gehrke T. Proceedings of the International Consensus Meeting on Periprosthetic Joint Infection. First ed. Maryland, United States: Data Trace Publishing Company, 2013.

[17] Stambough JB, Nam D, Warren DK, Keeney JA, Clohisy JC, Barrack RL, et al. Decreased hospital costs and surgical site infection incidence with a universal decolonization protocol in primary total joint arthroplasty. J Arthroplasty. 2017;32:728-734

[18] Weiser MC and Moucha CS. The current state of screening and decolonization for the prevention of staphylococcus aureus surgical site infection after total hip and knee arthroplasty. J Bone Joint Surg Am. 2015;97:1449-1458.

[19] Westberg M, Snorrason F, Frihagen F. Preoperative waiting time increased the risk of periprosthetic infection in patients with femoral neck fracture. Acta Orthop. 2013;84:124-129.

[20] Yoon RS, Mahure SA, Hutzler LH, Iorio R, Bosco JA. Hip arthroplasty for fracture vs elective care: one bundle does not fit all. J Arthroplasty. 2017;32:2353-2358 